\title{
INFLUENCE OF THERMAL RADIATION ON MAGNETOHYDRODYNAMIC (MHD) BOUNDARY LAYER FLOW OF A VISCOUS FLUID OVER AN EXPONENTIALLY STRETCHING SHEET
}

\author{
A.S. IDOWU* and S. USMAN \\ Department of Mathematics, University of Ilorin \\ Ilorin, NIGERIA \\ E-mails: asidowu@gmail.com; princemagee1@gmail.com
}

\begin{abstract}
Radiation on a magnetohydrodynamic (MHD) boundary layer flow of a viscous fluid over an exponentially stretching sheet was considered together with its effects. The new technique of homotopy analysis method (nHAM) was used to obtain the convergent series expressions for velocity and temperature, where the governig system of partial differential equations was transformed into ordinary differential equations. The interpretation of these expressions is shown physically through graphs. We observed that the effects of the Prandtl and magnetic number act in opposite to each other on the temperature.
\end{abstract}

Key words: boundary-layer, heat transfer, MHD, radiation, stretching sheet.

\section{Introduction}

In many engineering processes today an incompressible boundary layer flow due to an exponentially stretching sheet is useful in a good number of applications. Such applications include industrial manufacturing in the aerodynamic extrusion of plastic sheets, hot rolling, the boundary layer along a liquid film condensation process, cooling process of metal plate in a bath and in the polymer industries. It is seen that the kinematics of stretching with both the simultaneous heating or cooling during these processes has a great influence on the quality of end products (Magyari and Keller [1]). The work of Sakiadis [2] looked into the stretching flow problem. Crane [3] became the first to study the boundary layer flow caused by a stretching sheet which accelerates with a velocity varying linearly with the distance from a fixed point. Carragher and Crane [4] investigated the heat transfer area under this problem, with the conditions that the temperature difference between the surface and the ambient fluid is proportional to a power of the distance from a fixed point. The steady boundary layer on an exponentially stretching continuous surface with an exponential temperature distribution was also discussed by Magyari and Keller [1]. The effect of viscous dissipation on the mixed convection heat transfer from an exponentially stretching surface was studied by Partha et al. [5]. Hayat and Sajid [6] considered the radiation effects on the flow over an exponentially stretching sheet, where the problem was solved analytically using the homotopy analysis method. To deal with the problems such as cooling of nuclear reactors by liquid sodium and induction flow meter, which depends on the potential difference in the fluid in the direction perpendicular to the motion and to the magnetic field (Ganesan and Palani [7]), the MHD has important applications. Various processes in engineering areas occur at high temperature where radiation heat transfer becomes of great importance in the design of equipment (Seddeek [8]). Anuar [9] studied the MHD boundary layer flow on an exponentially stretching sheet taking the velocity gradient in the energy equation to be zero. The motivation to this present work is the variation of the velocity gradient in the problem of the MHD boundary layer flow over an exponentially stretching sheet in the presence of radiation where the velociy gradient in the energy equation is not zero, which has not been studied.

\footnotetext{
* To whom correspondence should be addressed
} 


\section{Problem formulation}

Consider a two-dimensional flow of an incompressible, steady viscous fluid bounded by a stretching sheet and conducted ellectrically which is placed in a fluid of uniform temperature $T_{\infty}$, given in Fig.1. with the magnetic field $B(x)$ applied normal to the sheet and the induced magnetic field neglected,which is justified for the MHD flow at small magnetic Raynold numbers. Under the usual boundary layer approximations, the flow and heat transfer with the radiation effects are governed by the following equations

$$
\begin{aligned}
& \frac{\partial u}{\partial x}+\frac{\partial v}{\partial y}=0, \\
& u \frac{\partial u}{\partial x}+v \frac{\partial u}{\partial y}=v \frac{\partial^{2} u}{\partial y^{2}}-\frac{\sigma B_{o}^{2} u}{\rho}, \\
& u \frac{\partial T}{\partial x}+v \frac{\partial T}{\partial y}=\frac{\kappa}{\rho C_{p}} \frac{\partial^{2} T}{\partial y^{2}}+\frac{\mu}{\rho C_{p}}\left(\frac{\partial u}{\partial y}\right)^{2}-\frac{1}{\rho C_{p}} \frac{\partial q_{r}}{\partial y}
\end{aligned}
$$

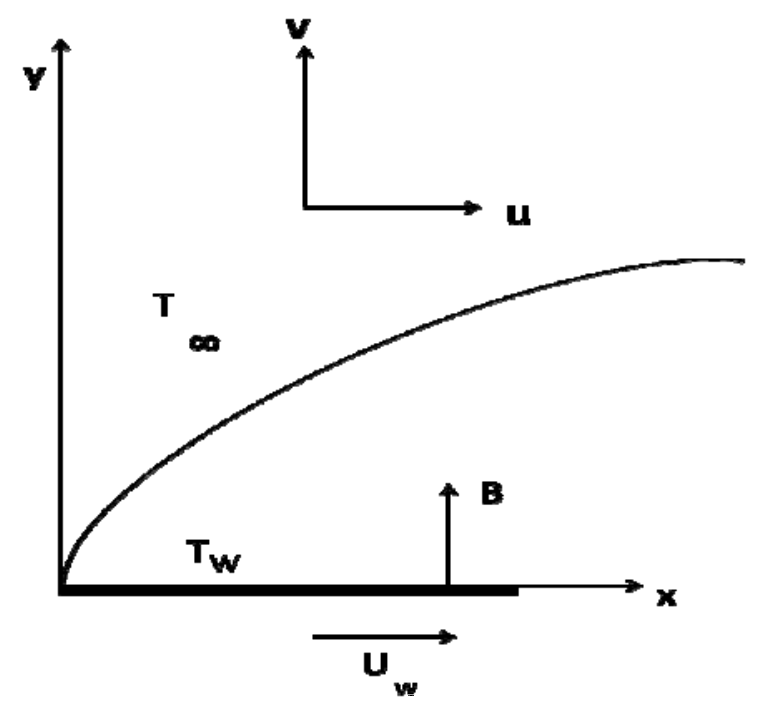

Fig.1. Physical model and coordinate system.

where $u$ and $v$ are the velocities in the $x$-and $y$-directions, respectively, $\rho$ is the fluid density, $v$ is the kinematic viscosity, $\mu$ is the dynamic viscosity, $\kappa$ the is thermal conductivity, $C_{p}$ is the specific heat, $T$ is the fluid temperature in the boundary layer and $q_{r}$ is the radiation heat flux.The boundary conditions are given by 


$$
\begin{aligned}
& u=U_{w}=U_{\circ} \exp \left(\frac{x}{l}\right), \quad v=0, \\
& T=T_{w}=T_{\infty}+T_{\circ} \exp \left(\frac{x}{2 l}\right) \quad \text { at } \quad y=0, \\
& u \rightarrow 0, \quad T \rightarrow T_{\infty} \quad \text { as } \quad y \rightarrow \infty
\end{aligned}
$$

where $U_{\circ}$ is the reference velocity, $T_{\circ}$ and $T_{\infty}$ are, respectively, the temperature at the plate and far from the plate and $L$ is the reference length. A derivation of reasonable simplifications is required to understand fluid radiation (Aboeldahab and El Gendy [10]). One of these simplifications was made by Cogley et al. [11] who assumed that the fluid does not absorb its own radiation,but per perticle it only absorbs radiation emitted by the boundaries.Hence, the problem can be simplified by using the Rosseland approximation (Rosseland [12]; Siegel and Howell [13] Sparrow and Cess [14]) which simplifies the radiation heat flux as

$$
q_{r}=-\frac{4 \sigma^{*}}{3 \kappa^{*}} \frac{\partial T^{4}}{\partial y}
$$

where $\sigma^{*}$ and $\kappa^{*}$ are the Stefan-Boltzmann constant and the mean absorption coefficient, respectively.This approximation is valid at points optically far from the boundary surface and it is good only for intensive absorption which is far, for an optically thick boundary layer (Bataller [16]; Siegel and Howell [13];Sparrow and Cess [14], Raptis [15]. Assuming that the temperature differences within the flow such that the term $T^{4}$ may be expressed as a linear function of temperature.Expanding $T^{4}$ in a Taylor series about $T_{\infty}$ and neglecting higher order terms gives

$$
T^{4} \approx 4 T_{\infty}^{3} T-3 T_{\infty}^{4}
$$

Using Eqs (2.5) and (2.6), Eq.(2.3) reduces to

$$
u \frac{\partial T}{\partial x}+v \frac{\partial T}{\partial y}=\frac{k}{\rho C_{p}} \frac{\partial^{2} T}{\partial y^{2}}+\frac{\mu}{\rho C_{p}}\left(\frac{\partial u}{\partial y}\right)^{2}+\frac{16 T_{\infty}^{3} \sigma^{*}}{\rho C_{p} 3 k^{*}} \frac{\partial^{2} T}{\partial y^{2}} .
$$

To get the similarity solutions, we assumed that the magnetic field $B(x)$ is of the form

$$
B=B_{\circ} \exp \left(\frac{x}{2 L}\right)
$$

where $B$ is the constant magnetic field.

Equation (2.1) is satisfied by introducing a stream function $\psi$ such that 


$$
u=\frac{\partial \psi}{\partial y} \quad \text { and } \quad v=-\frac{\partial \psi}{\partial x}
$$

Equations (2.2) and (2.3) are transformed into the corresponding ordinary differential equations by the following transformation(Hayat and Sajid [6])

$$
\begin{aligned}
& u=U_{\circ} \exp \left(\frac{x}{L}\right) f^{\prime}(\eta), \quad v=-\left(\frac{v U_{\circ}}{2 L}\right)^{\frac{1}{2}} \exp \left(\frac{x}{2 L}\right)\left(f(\eta)+\eta f^{\prime}(\eta)\right), \\
& T=T_{\infty}+T_{\circ} \exp \left(\frac{x}{2 L}\right) \theta(\eta), \quad \eta=\left(\frac{U_{\circ}}{2 v L}\right)^{\frac{1}{2}} \exp \left(\frac{x}{2 L}\right) y
\end{aligned}
$$

where $\eta$ is the similarity variable, $f(\eta)$ is the dimensionless stream function, $\theta(\eta)$ is the dimensionless temperature and prime denotes differentiation with respect to $\eta$. The transformed ordinary differential equations are

$$
\begin{aligned}
& f^{\prime \prime \prime}+f f^{\prime \prime}-2 f^{\prime 2}-M f^{\prime}=0, \\
& {\left[1+\frac{4 K}{3}\right] \theta^{\prime \prime}(\eta)+\mathrm{P}_{\mathrm{r}}\left[\theta^{\prime}(\eta) f(\eta)-\theta(\eta) f^{\prime}(\eta)+E f^{\prime \prime 2}(\eta)\right]=0} \\
& \text { where } \quad M=\frac{2 \sigma B_{\circ}^{2} L}{\rho U_{\circ}}, \quad K=\frac{4 \sigma^{*} T_{\infty}^{3}}{k k^{*}}, \quad \mathrm{P}_{\mathrm{r}}=\frac{\mu C_{p}}{k} \quad \text { and } \quad E=\frac{U_{\circ}^{2}}{T_{\circ} C_{p}},
\end{aligned}
$$

are the magnetic parameter, radiation parameter, Prandtl parameter and Eckert parameter respectively. The transformed boundary conditions are

$$
\begin{aligned}
& f(0)=0, \quad f^{\prime}(0)=1, \quad \theta(0)=1, \\
& f^{\prime}(\eta) \rightarrow 0, \quad \theta(\eta) \rightarrow 0 \quad \text { as } \quad \eta \rightarrow \infty .
\end{aligned}
$$

\section{3. nHAM solution}

In order to solve Eqs (2.11)-(2.13) using nHAM, assume that $f^{\prime \prime}(0)=\alpha$ and $\theta^{\prime}(0)=\beta$. We construct a system of differential equations as follows

$$
\begin{aligned}
& f^{\prime}(\eta)=v, \\
& v^{\prime}(\eta)=w, \\
& w^{\prime}(\eta)=2 f^{\prime 2}-f f^{\prime \prime}+M f^{\prime},
\end{aligned}
$$


with intial approximations

$$
f_{\circ}(\eta)=0, \quad v_{\circ}(\eta)=1, \quad w_{\circ}(\eta)=\alpha,
$$

the auxiliary linear operators are $L f(\eta)=\frac{\partial f}{\partial \eta}, L v(\eta)=\frac{\partial v}{\partial \eta}, L w(\eta)=\frac{\partial w}{\partial \eta}$ where $L$ is an auxiliary linear operator, and

$$
\begin{aligned}
& \theta^{\prime}(\eta)=C, \\
& C^{\prime}(\eta)=-\frac{\mathrm{P}_{\mathrm{r}}}{1+\frac{4 K}{3}}\left[f \theta^{\prime}-f^{\prime} \theta+E f^{\prime \prime 2}\right],
\end{aligned}
$$

using initial approximations

$$
\theta_{\circ}(\eta)=1, \quad C_{\circ}(\eta)=\beta,
$$

and the auxiliary linear operators are

$$
L \theta(\eta)=\frac{\partial \theta}{\partial \eta}, \quad L C(\eta)=\frac{\partial C}{\partial \eta}
$$

we then have

$$
\begin{aligned}
& f_{l}(\eta)=\hbar_{1} \int_{0}^{\eta}\left[-v_{\circ}(\eta)\right] d \eta, \\
& v_{l}(\eta)=\hbar_{1} \int_{0}^{\eta}\left[-w_{\circ}(\eta)\right] d \eta, \\
& w_{l}(\eta)=\hbar_{1} \int_{0}^{\eta}\left[-M v_{\circ}-2 v_{\circ}^{2}+f_{\circ} w_{\circ}\right] d \eta,
\end{aligned}
$$

and

$$
\begin{aligned}
& \theta_{1}(\eta)=\hbar_{2} \int_{0}^{\eta}\left[-C_{\circ}(\eta)\right] d \eta, \\
& C_{1}(\eta)=\frac{\mathrm{P}_{\mathrm{r}} \hbar_{2}}{1+\frac{4 K}{3}} \int_{0}^{\eta}\left[f_{\circ} C_{\circ}-f_{\circ}^{\prime} \theta_{\circ}+E f_{\circ}^{\prime \prime 2}\right] .
\end{aligned}
$$

For $m \geq 2$, 


$$
\begin{aligned}
& f_{m}(\eta)=\left(1+\hbar_{1}\right) f_{m-1}(\eta)+\hbar_{1} \int_{0}^{\eta}\left[-v_{m-1}(\eta)\right] d \eta \\
& v_{m}(\eta)=\left(1+\hbar_{1}\right) v_{m-1}(\eta)+\hbar_{1} \int_{0}^{\eta}\left[-w_{m-1}(\eta)\right] d \eta \\
& w_{m}(\eta)=\left(1+\hbar_{1}\right) w_{m-1}(\eta)+\hbar_{1} \int_{0}^{\eta}\left[-M f_{m-1}^{\prime}+\sum_{i=0}^{m-1}\left(-2 f_{m-1-i}^{\prime} f_{i}^{\prime}+f_{m-1-i} f_{i}^{\prime \prime}\right)\right] d \eta
\end{aligned}
$$

and

$$
\begin{aligned}
& \theta_{m}(\eta)=\left(1+\hbar_{2}\right) \theta_{m-1}(\eta)+\hbar_{2} \int_{0}^{\eta}\left[-C_{m-1}(\eta)\right] d \eta, \\
& C_{m}(\eta)=\left(1+\hbar_{2}\right) C_{m-1}(\eta)+\frac{\mathrm{P}_{\mathrm{r}} \hbar_{2}}{1+\frac{4 K}{3}} \int_{0}^{\eta} \sum_{i=0}^{m-1}\left[f_{m-1-i} \theta_{i}^{\prime}-f_{m-1-i}^{\prime} \theta_{i}+E f_{m-1-i}^{\prime \prime} f_{i}^{\prime}\right] d \eta
\end{aligned}
$$

The systems of Eqs.(3.5)-(3.8) have been solved using the symbolic computation software MAPLE. It is found that

$$
\begin{aligned}
& f_{1}(\eta)=-\hbar_{1} \eta, \\
& v_{1}(\eta)=-\hbar_{1} \alpha \eta, \\
& w_{1}(\eta)=\hbar_{1}(-2 \eta-M \eta), \\
& f_{2}(\eta)=-\left(1+\hbar_{1}\right) \hbar_{1} \eta+\frac{1}{2} \hbar_{1}^{2} \alpha \eta^{2}, \\
& v_{2}(\eta)=-\left(1+\hbar_{1}\right) \hbar_{1} \alpha \eta-\frac{1}{2} \hbar_{1}^{2}(-2-M) \eta^{2}, \\
& w_{2}(\eta)=\left(1+\hbar_{1}\right) \hbar_{1}(-2 \eta-M \eta)+\frac{1}{2}\left(M \hbar_{1} \alpha+3 \hbar_{1} \alpha\right) \eta^{2},
\end{aligned}
$$

and

$$
\begin{aligned}
& \theta_{1}(\eta)=-\hbar_{2} \beta \eta, \\
& C_{l}(\eta)=-\frac{\mathrm{P}_{\mathrm{r}} \hbar_{2} \eta}{1+\frac{4 K}{3}},
\end{aligned}
$$




$$
\begin{aligned}
& \theta_{2}(\eta)=-\left(1+\hbar_{2}\right) \hbar_{2} \eta \beta+\frac{1}{2} \frac{\hbar_{2}^{2} \mathrm{P}_{\mathrm{r}} \eta^{2}}{1+\frac{4 K}{3}}, \\
& C_{2}(\eta)=-\frac{\left(1+\hbar_{2}\right) \mathrm{P}_{\mathrm{r}} \hbar_{2} \eta}{1+\frac{4 K}{3}}+\frac{0.50 \mathrm{P}_{\mathrm{r}} \hbar_{2}\left(0.70 \beta+\hbar_{2} \beta\right) \eta^{2}}{1+\frac{4 K}{3}} .
\end{aligned}
$$

$f_{m}\left(\eta, \alpha ; \hbar_{1}\right)(m=3,4,5, \ldots)$ and $\theta_{2}\left(\eta, \beta ; \hbar_{2}\right)(m=3,4,5, \ldots)$ can be calculated similarly. Then the series solution expressions by nHAM can be written in the form

$$
\begin{aligned}
& F_{Q}\left(\eta, \alpha, \hbar_{1}\right)=\sum_{m=0}^{Q} f_{m}\left(\eta, \alpha, \hbar_{1}\right) \\
& \theta_{N}\left(\eta, \beta, \hbar_{2}\right)=\sum_{m=0}^{N} \theta_{m}\left(\eta, \beta, \hbar_{2}\right),
\end{aligned}
$$

we note that the analytic expressions (3.13) and (3.14) contain two auxiliary parameters $\hbar_{1}$, and $\hbar_{2}$ as suggested by Liao [17] and [18]; (Bidin and Nazar[19]; Hany and Magdy [20] and [21]). Choose the values of $\hbar_{1}$ , and $\hbar_{2}$ properly from $\hbar$-curves which ensure the convergence of the series solutions.Using the boundary condition $f^{\prime} \rightarrow 0$ as $\eta \rightarrow \infty$ and $\theta \rightarrow 0$ as $\eta \rightarrow \infty$ we get $\alpha=0$ for $M=0$ and also $\beta=0$ for $K=0, M=0, \mathrm{P}_{\mathrm{r}}=0, E=0$.

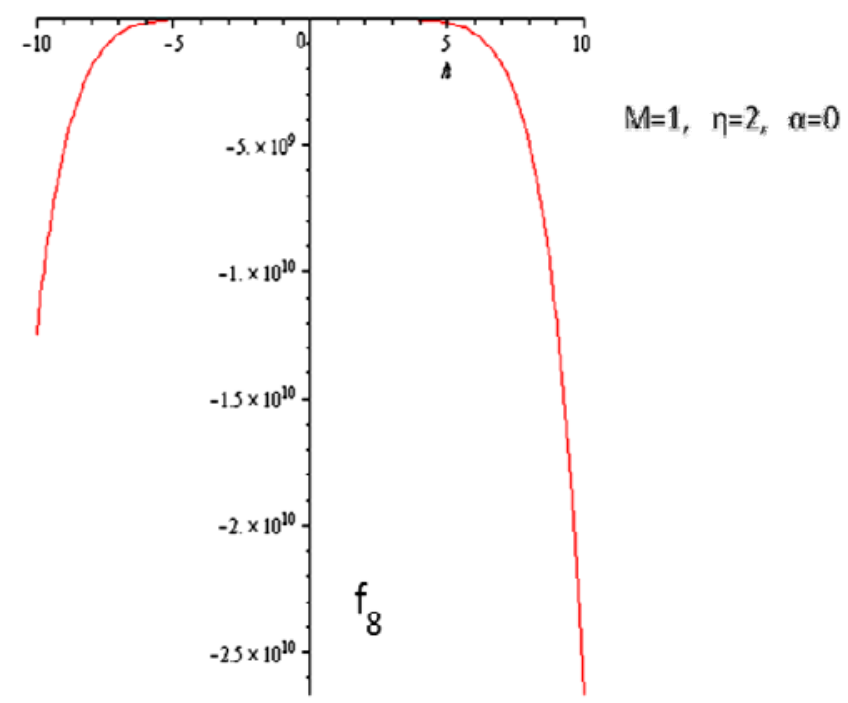

Fig.2. The $\hbar_{2}$ curve. 


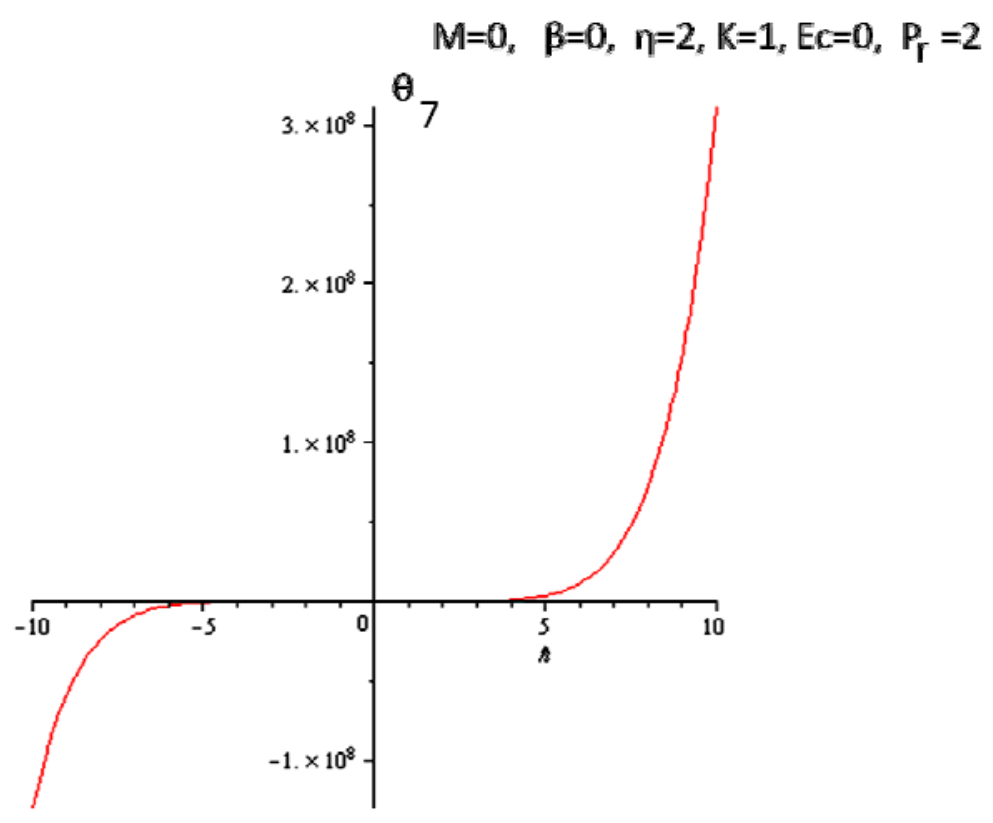

Fig.3. The $\hbar_{2}$ curve.

In Figs 2 and 3 the $\hbar$-curves are shown for the range of admissible values of $\hbar_{1}$, and $\hbar_{2}$. Figures 2 and 3 clearly indicate that the ranges for the admissible values of $\hbar_{1}$, and $\hbar_{2}$ are $[-5,6,4,2]$ and $[-5,1,3,1]$.

Our calculations shows that the series solution (3.13) and (3.14) converge in the whole region of $\eta$, when $\hbar_{1}=-0.7, \hbar_{2}=-1$.

\section{Results and discusion}

The system of ordinary differential Eqs (2.11)-(2.13) have been solved numerically using nHAM as described by Hassan and El-Tawil [22]. This method has been used to solve several boundary layer problems. We show the graphical results of velocity and temperature. Attention has been focused on the variations of $\operatorname{Pr}, M$, E and $K$. For this purpose Figs 4-8 have been displayed. Figure 4 shows the effect of the magnetic number on the velocity $f^{\prime}(\eta)$. Figures 5-8 elucidate the influence of the radiation number $K$, magnetic number $M$, Prandtl number Pr and Eckert number Ec on the temperature $\theta(\eta)$. From the present study, the main findings can be summarized as follows:

- Increase in the magnetic parameter $M$ has an accelerating effect on the velocity of the flow field.

- Increase in the radiation parameter $K$, magnetic parameter $M$ and Eckert number Ec retards the magnitude of temperature of the flow field and the thickness of the thermal boundary layer.

- Increase in the Prandtl number Pr shows that there is a rise in the magnitude of temperature of the flow field and the thickness of the thermal boundary layer. 


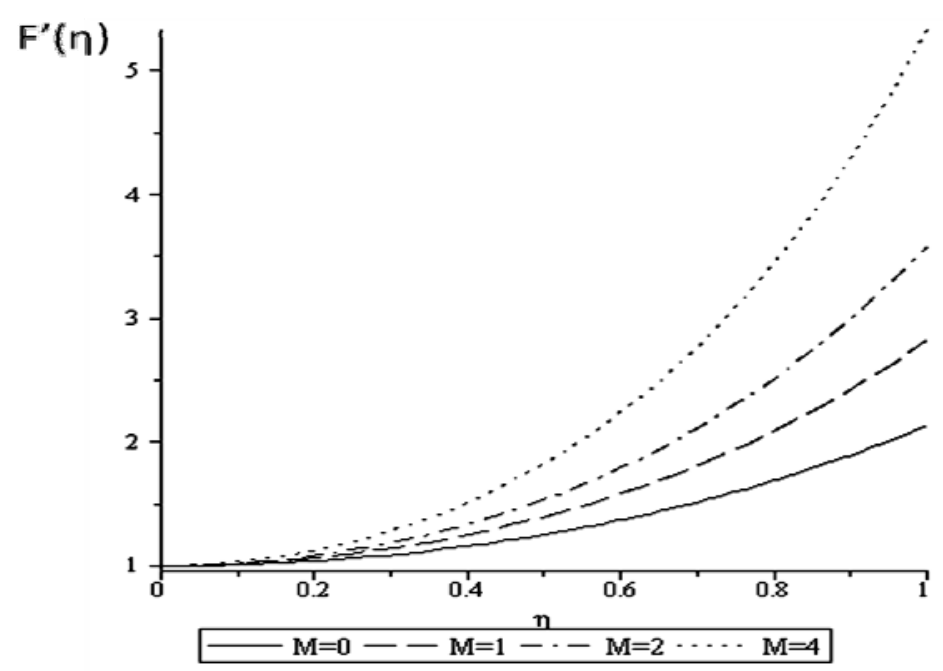

Fig.4. The effect of the magnetic parameter, $M$ on velocity, $f^{\prime}$ for $\alpha=0, \hbar=-0.7$.

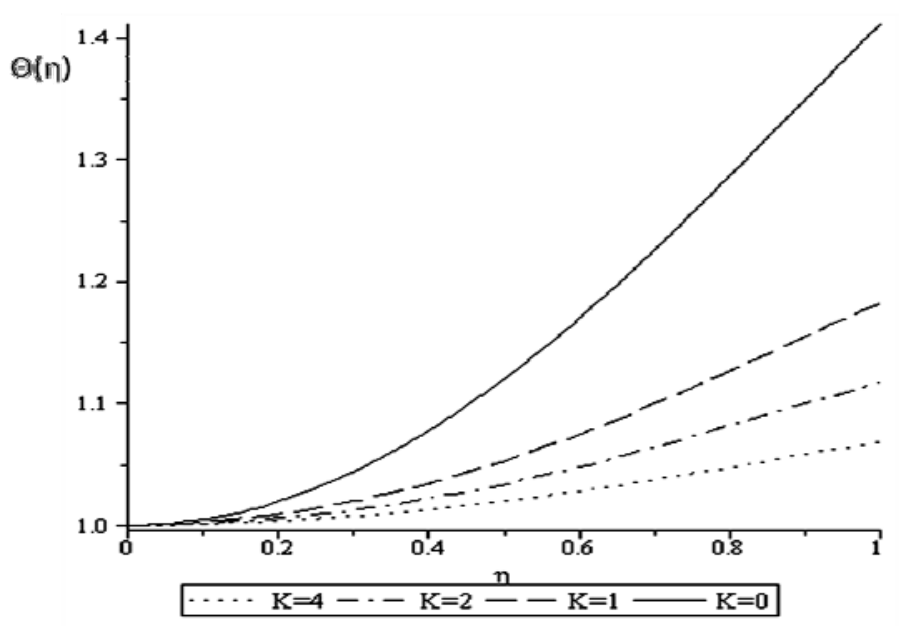

Fig.5. The effect of the radiation parameter, $K$ on temperature, $\theta$ for $\operatorname{Pr}=1, \mathrm{Ec}=0.2, M=1$.

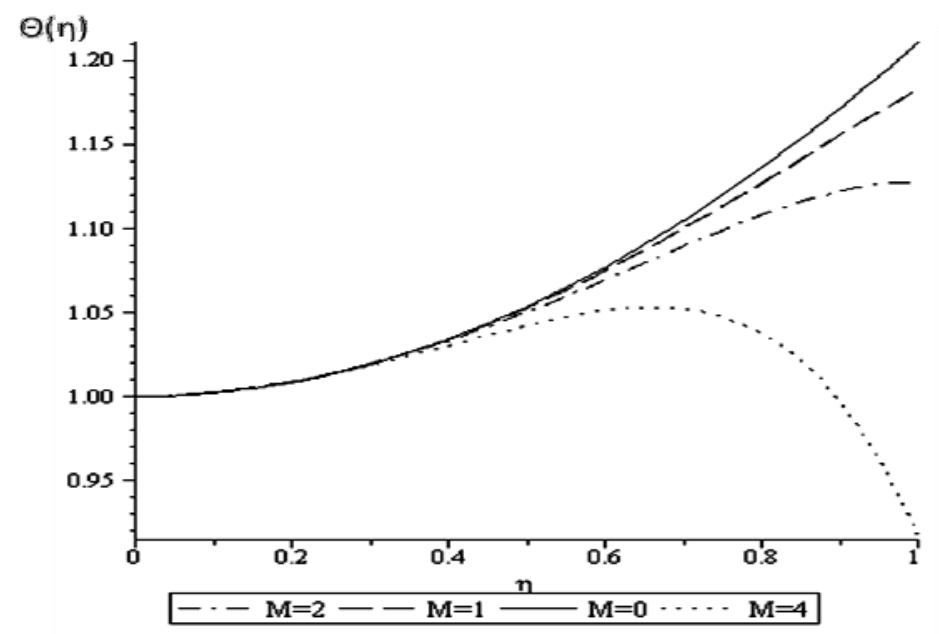

Fig.6. The effect of the magnetic parameter, $M$ on temperature, $\theta$ for $\operatorname{Pr}=1, \mathrm{Ec}=0.2, K=1$. 


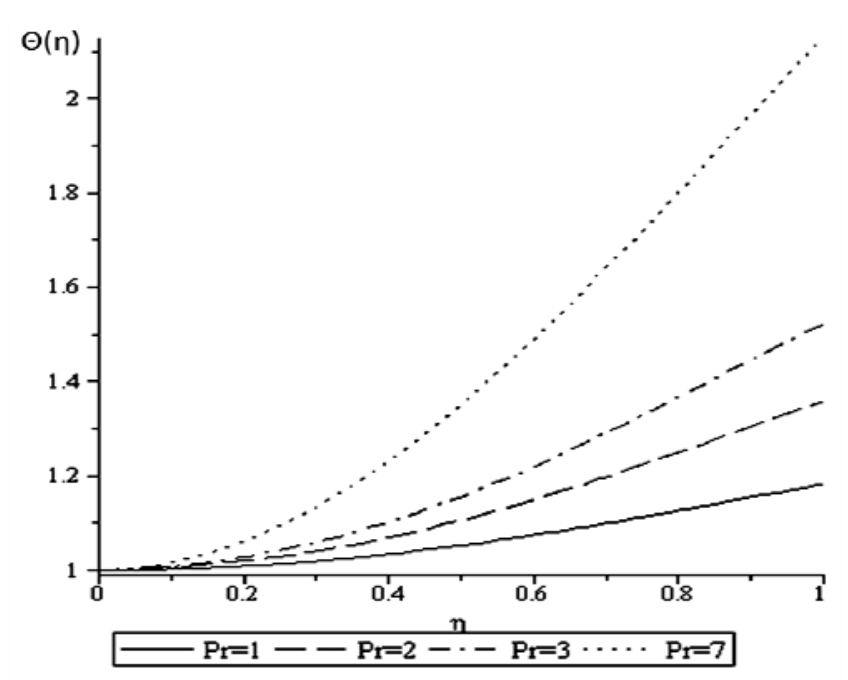

Fig.7. The effect of the Prandtl number, Pr on temperature, $\theta$ for $K=1, \mathrm{Ec}=0.2, M=1$.

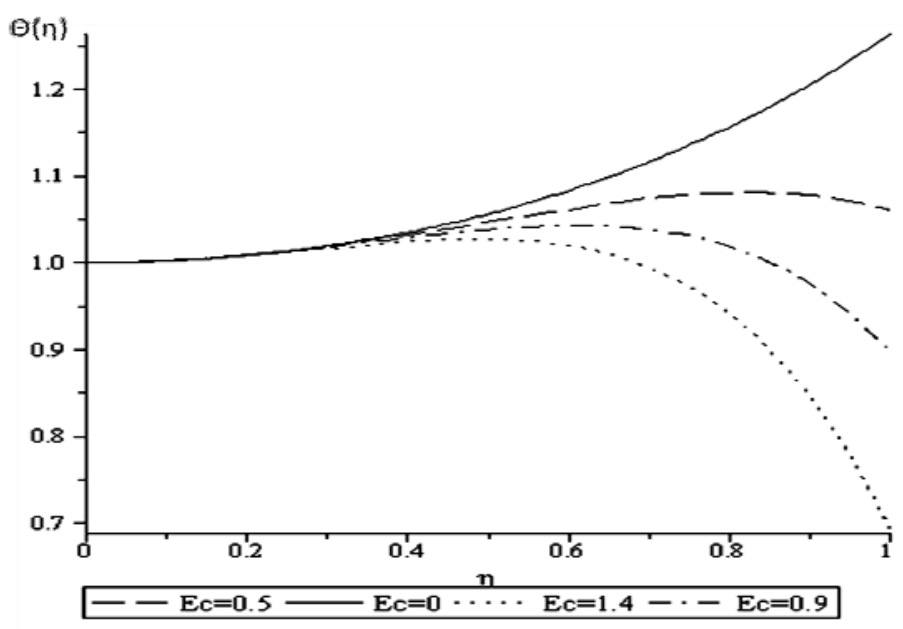

Fig.8. The effect of the Eckert number, Ec on temperature, $\theta$ for $K=1, \mathrm{P}_{\mathrm{r}}=1, M=1$.

\section{Conclusions}

Radiation on a steady MHD boundary layer flow over an exponentially stretching sheet was investigated and its effects observed. The similarity transformations are used to reduce the partial differential equations into ordinary differential equations. Analytical solutions for the velocity and temperature distributions are obtained using an nHAM. It was found that the heat rate increases with the Prandtl number $\operatorname{Pr}$, but decreases with both the magnetic parameter $M$ and radiation parameter $K$. Thus the magnetic and radiation parameter brought about the cooling effect on the sheet, because a higher the increase in the parameter reduces the heat rate.

\section{Nomenclature}

$$
\begin{aligned}
& B_{\circ}-\text { constant magnetic field } \\
& C_{p}-\text { specific heat }
\end{aligned}
$$




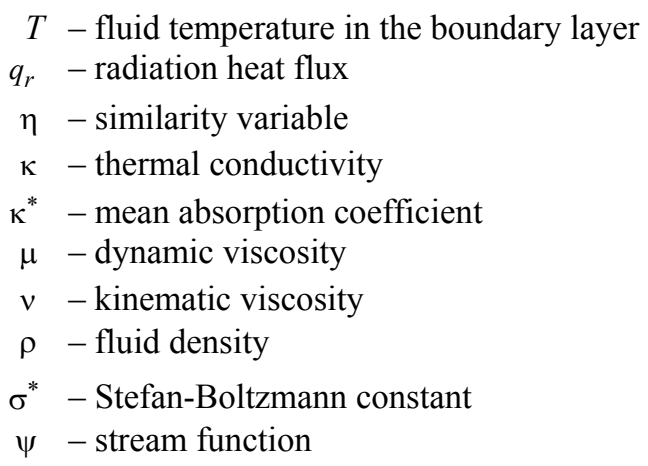

\section{References}

[1] Magyari E. and Keller B. (1999): Heat and transfer in the boundary layer on an exponentially stretching continuous surface. - J. Phys. D. Appl. Phys., vol.32, pp.577-585.

[2] Sakiadis B.C. (1961): Boundary layer bahaviour on continous solid surface: I boundary layer equation for two dimensional and axisymmetric flow. - AIChE J., vol.7, pp.26-28.

[3] Crane, L.J. (1970): Flow past a stretching plate. - Z. Angew. Math. Mech., vol.21, pp.645-647.

[4] Carragher P. and Crane L.J. (1982): Heat transfer on a continuous streeting sheet. - Z. Angew. Math. Mech., vol.62, 564-573.

[5] Partha M.K., Murthy P.V.S.N. and Rajasekhar G.P. (2005): Effect of viscous dissipation on the mixed convection heat transfer from an exponentially stretching surface. - Heat Mass Transfer, vol.41, pp.360-366.

[6] Hayat T. and Sajid M. (2008) Analytic solution for axisymmetric flow and heat transfer of a second grade fluid past a stretching sheet. - Int. J. Heat Mass Transfer, vol.50, pp.75-84.

[7] Ganesan P. and Palani G. (2004): Finite difference analysis of unsteady natural convection MHD past an inclined plate with variable surface heat and mass flux. - Int. J. Heat Mass Transfer, vol.47, pp.4449-4457

[8] Seddeed M.A. (2002): Effect of radiation and variable viscousity on a MHD free convection flow past a semiinfinite flat plate with an aligned magnetic field in the case of unsteady flow. -Int. J. Heat Mass Transfer, vol.45, pp.931-935.

[9] Anuar I. (2011): MHD boundary layer flow due to an exponentially stretching sheet with radiation effect. - Sains Malaysiana, vol.40, No.4, pp.391-395.

[10] Aboeldahab E.M. and El Gendy M.S. (2002): Radiation effect on MHD free convective flow of a gas past a semiinfinite vertical plate with variable thermos-physical properties for high-temperature differences. - Can. J. Phys., vol.80, pp.1609-1619.

[11] Cogley A.C., Vincenty W.G. and Gilles S.E. (1968): Differential approximation for radiative transfer in a nongrey gas near equilibrium. - AIAA J., vol.6, pp.551-553.

[12] Rosseland S. (1936): Theoretical Astrophysics. - New York: Oxford University.

[13] Siegel R. and Howell J.R. (1992): Thermal Radiation: Heat Transfer 3rd ed. - Washington DC: Hemisphere.

[14] Sparrow E.M. and Cess R.D. (1978): Radiation Heat Transfer. -Washinton DC: Hemisphere.

[15] Raptis A. (2004): Effect of thermal radiationon MHD flow. - Appl. Math. Comput., vol.32, pp.577-585.

[16] Bataller R.C. (2008): Similarity solutions for boundary layer flow and heat transfer of a FENE-P fluid with thermal radiation. - Phys. Lett.A, vol.372, pp.2431-2439.

[17] Liao S.J. (1992): The proposed Homotopy Analysis Technique for the Solution of Nonliear Problems. - Ph.D. Thesis. 
[18] Liao S.J. (2003): Beyond Perturbation:Introduction to Homotopy Analysis Method. - Chapman and Hall/CRC Press.

[19] Bidin B. and Nazar R. (2009): Numerical solution of the boundary Layer Flow over an Exponentially Stretching Sheet with Thermal Radiation.Euro.J.Sci. 33(4) : $710-717$.

[20] Hany N.H. and Magdy A.E. (2012): A new technique of using homotopy analysis method for second order nonlinear differential equation. - Appl. Math. Comput., vol.219, pp.708-728.

[21] Hany N.H. and Magdy A.E. (2010): A New Technique of Using Homotopy Analysis Method for Solving HighOrder Nonlinear Differential Equations. - Wiley online Libery.

[22] Hassan H.N. and El-Tawil M.A. (2012): A new technique of using homotopy analysis method for second order nonlinear differential equations. - Applied Mathematics and Computation, vol.219, No.2, pp.708-728.

Received: March 11, 2015

Revised: May 9, 2016 